CHAPTER VI

MEMBERSHIP 


\section{1) LIST OF ADHERING COUNTRIES}

The year of adherence and approximate number of IAU members in the geographical area are indicated (as of May 1986).

Country

Arab Republic of Egypt

Argentina

Australia

Austria

Belgium

Brazil

Bulgaria

Canada

Chile
Adhering Organization

Academy of Scientific Research \& Technology Dept. of Scientific Societies

and International Unions

lol Kasr El-Einy Street

Cairo

Consejo Nacional de Investigaciones

1927

57

Cientificas y Técnicas

Rivadavia 1917

1033 Buenos Aires

Australian Academy of Sciences

1939

PO Box 783

Canberra City, ACT 2601

Osterreichische Akademie der Wissenschaften

Dr. Ignaz-Seipel-Platz 2

A-1010 Wien

Académie Royale de Belgique

1920

Palais des Académies

Rue Ducale 1

B-1000 Bruxelles

1955

32

Conselho Nacional de Desenvolvimento

1961

39

Cientifico e Tecnologico - CNPq

Av. W3 Norte, Quadra 507-B

Caixa Postal 11-1142

70740 Brasilia DF

Bulgarian Academy of Sciences

1957

37

7 th November Street 1

1000 Sofia

National Research Council of Canada

International Relations

Ottawa, Ontario KIA OR6

1957

189

Universidad de Chile

1947

Observatorio Astronomico Nacional

Casilla 36-D

Santiago 


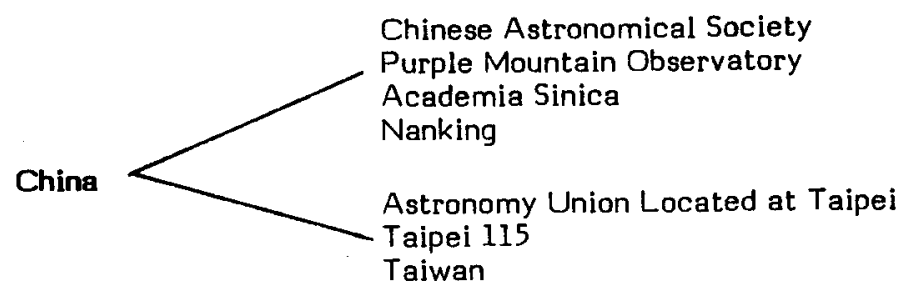

Colombia

Cuba

Czechoslovakia

Denmark

Finland

France

Germany, DR

Germany, FR

Greece

Hungary

India
Universidad Nacional de Colombia

1967 Apartado Aereo No. 5997

Bogota

Academia de Ciencias de Cuba

1970

Capitolio Nacional

La Habana 2

Czechoslovak Academy of Sciences

Narodni 3

Praha 1

Kongelige Danske Videnskabernes

1922

48

Selskab

H.C. Andersen Boulevard 35

DK-1553 København V

Académie des Sciences et Lettres

1948

Snellmaninkatu 9-11

Helsinki 17

Académie des Sciences

1920

414

COFUSI

23, quai Conti

F-75006 Paris

Akademie der Wissenschaften der DDR

1951

46

DDR-108 Berlin

Otto-Nuschke-Strasse 22/23

Rat Westdeutscher Sternwarte

1951

278

MPI für Physik \& Astrophysik

Karl-Schwarzschild-str. 1

D-8046 Garching bei München

Academy of Athens

1920

67

Panepistimiou Str.

Athens

Hungarian Academy of Sciences

1947

33

Roosevelt Tér 9

Budapest $V$

Indian National Science Academy

Bahadur Shah Zafar Marg

New Delhi 110002 
Indonesia

Iran

Iraq

Ireland

Israel

Italy

Japan

Korea, DPR

Korea, Republic of

Mexico

Netherlands

New Zealand
Indonesian Institute of Sciences (LIPI)

1979

4

Gedung Widya Graha

J1. Jend. Gatot Subroto 10

Jakarta Selatan

Geophysics Institute of Tehran University

1969

10

North Amir-Abad Avenue

Tehran

Council for Scientific Research

1976

10

Astronomy and Space Research Center

PO Box 255

Baghdad

The Royal Irish Academy

1947

Academy House

19 Dawson Street

Dublin 2

The Israel Academy of Sciences and Humanities

1954

37

Albert Einstein Square, Talbieh

Jerusalem 91040

Consiglio Nazionale delle Ricerche

1920

302

Piazzale Aldo Moro 7

I-00100 Roma

Science Council of Japan

1920

282

22-34 Roppongi 7 chome

Minato-Ku

Tokyo 106

Academy of Sciences of DPR of Korea 1961

21

Pyongyang

Korean National Astronomical Observatory

1973

Yoksam-Dong

Kangnam-ku

Seoul, 134-03

Instituto de Astronomia, UNAM

1921

43

Apartado Postal 70-264

Ciudad Universitaria

04510 DF Mexico

Koninklijke Nederlandse Akademie

1922

121

van Wetenschappen

Kloveniersburgwal 29

NL-1011 JV Amsterdam

The Royal Society of New Zealand

PO Box 12249

1964

22

Wellington 
Nigeria

Norway

Poland

Portugal

Roumania

South Africa

Spain

Sweden

Switzerland

Turkey

United Kingdom
Nigerian Academy of Sciences

Faculty of Science, University of Lagos

P.M.B. 1004

University of Lagos Post Office

Lagos

Det Norske Videnskaps-Akademi i Oslo

1922

21

Drammensveien 78

Oslo 2

Polskiej Akademii Nauk

1922

82

Palac Kultury i Nauki

Skrytka pocztowa 24

00-901 Warszawa

Secçao Portuguesa das Unioes

1924

16

Internacionais Astronomica e Geodesica e

Geofisica

Praça de Estrela

Lisboa 1200

Roumanian National Committee of Astronomy

1928

16

Astronomical Observatory

Cutitul de Argint 5

PO Box 28

75212 Bucarest

Council for Scientific and Industrial Research

1938

22

International Relations Division

PO Box 395

Pretoria 0001

Comision Nacional de Astronomia

1922

73

Instituto Geografico y Cadastral

General Ibanez 3

Madrid (3)

Kungl. Vetenskapsakademien

1925

73

OO Box 50005

S-184 05 Stockholm 50

Schweizerische Naturforschende Gesellschaft

1923

53

Zentralsekretariat

Postfach 2535

Hirschengraben 11

$\mathrm{CH}-3001$ Bern

Astronomi Denergi Baskani

1961

47

Universitet Rasthanesi

Universite

Istanbul

The Royal Society

6, Carlton House Terrace

London SWIY 5AG 
Uruguay

USA

USSR

Vatican City State

Venezuela

Yugoslavia
Ministerio de Relaciones Exteriores

1970

1

Avenida 18 de Julio 1205

Montevideo

National Academy of Sciences

1920

1615

Office of International Affairs 2101 Constitution Avenue N.W. Washington, DC 20418

Academy of Sciences of the USSR

1935

420

Foreign Relations Department

Leninskij Prospekt 14

Moscow 71

Pontificia Academia delle Scienze

1932

7 I-00120 Citta del Vaticano

Comité Nacional de Astronomia

1953

8

CIDA

Apartado 264

Mérida 5101-A

Savez Drustava Matematicara, Fizicara i

1935

31

Astronoma Jugosloslavije

Institut za Matematiku i Fiziku

Cetinjski put bb

81000 Titograd 